\title{
COARSE UNIFORMITIES ON THE RATIONALS ${ }^{1}$
}

\author{
H. C. ENOS
}

\begin{abstract}
There exist uniform spaces $\mu Q$ homeomorphic with the space $Q$ of rational numbers such that every homeomorphic uniform space admits a I liformly continuous homeomorphism upon $\mu Q$. If "homeomorphism" is replaced by "bijection", the resulting weaker property is equivalent to having as completion a Peano continuum. With "homeomorphism", a (countable) dense subspace of a Hilbert cube has the property, but not a dense subspace of an interval.
\end{abstract}

Introduction. One can, in a sense, complete these results by describing all uniformities $\mu$ on $Q$ such that every homeomorphic uniform space admits a uniformly continuous homeomorphism upon $\mu Q$ as follows: they are those whose completion is a quotient space of a Hilbert cube by a mapping under which the inverse image of each point of $Q$ is a single point. But nothing is known about such quotient spaces except that (of course) they are Peano continua, and their local cut points form a set of the first category.

The results extend investigations of Mrowka [3], who showed among other things that a countable metric space admits a uniformly continuous homeomorphism into a Hilbert cube. Mrowka asked whether a countable dense-in-itself subspace of an interval $I$ can always be mapped bijectively to a dense subspace of $I$ by a continuous mapping $I \rightarrow I$. It can, by the main result here on uniformly continuous bijections (Theorem 3 below, using also Theorem 1; we get the mapping by way of a Hilbert cube). Mrowka asked also whether the plane admits a continuous mapping into $I$ whose restriction to a dense subset is homeomorphic. The result on local cut points (Theorem 2) shows that it does not.

I am indebted to the referee for replacing part of my proof with a reference to Fort [1].

1. Compatible case. Each uniformity $\mu$ on $Q$ (or any other space) contains a precompact uniformity $v$, still compatible with the topology.

Received by the editors August 17, 1971.

AMS 1970 subject classifications. Primary 54E45; Secondary 54E15.

Key words and phrases. Uniformity, quotient space.

${ }^{1}$ Work supported by National Science Foundation grant GP-16951. 
In turn $v$ contains a compatible metric uniformity $\pi[2$, p. 106], which is still precompact. So $\pi$ is induced by an embedding of $Q$ in a Hilbert cube $H$.

LEMMA. Every subset of $H$ nomeomorphic with $Q$ admits a uniformly continuous dense topological embedding into $H$.

Proof. The peculiar property of $H$ that we need is this: each point has a basis of closed neighborhoods, homeomorphic with $H$, which admit continuous retractions upon their boundaries. That is evident for a geometric extreme point. It is true for all points since $H$ is homogeneous [1].

Call the given subset $Q=\left\{q_{1}, q_{2}, \cdots\right\}$. Its closure $C$ is a perfect metric space and therefore admits a continuous mapping $f_{0}$ onto $H$. We construct mappings $f_{n}: C \rightarrow H$ such that the first $n$ points $q_{i}$ of $Q$ are inverse images $f_{n}^{-1} f_{n}\left(q_{i}\right)$. Having $f_{n}$, the point $p_{n}=f_{n}\left(q_{n+1}\right)$ differs from the previous $f_{n}\left(q_{i}\right)$. Let $\left\{N_{n j}\right\}$ be a sequence of neighborhoods of $p_{n}$, shrinking to $p_{n}$, admitting continuous retractions $r_{n j}$ upon their boundaries. We want $N_{n 1}$ small, but we delay (till $(\alpha)$ and $(\beta)$ below) specifying how small. Choose a neighborhood $M_{1}$ of $q_{n+1}$ remote from $q_{1}, \cdots, q_{n}$ and interior to $f_{n}^{-1}\left(N_{n 1}\right)$. The first modification $s_{1}$ of $f_{n}$ is to satisfy the following conditions. On the complement of $f_{n}^{-1}\left(N_{n 1}\right), s_{1}$ agrees with $f_{n}$; on $f_{n}^{-1}\left(N_{n 1}\right)-M_{1}, s_{1}$ is $r_{n 1} f_{n}$; $s_{1}\left(M_{1}\right)=N_{n 1}$, and $s_{1}\left(q_{n+1}\right)=p_{n}$. Such a continuous mapping exists because $N_{n 1}$ is an injective compact space and $C$ is perfect. Observe that $s_{1}$ is onto; for the image contains $N_{n 1}$, and $s_{1}$ agrees with $f_{n}$ outside $f_{n}^{-1}\left(N_{n 1}\right)$. Then for a basis of neighborhoods $M_{j} \subset s_{j-1}^{-1}\left(N_{n j}\right)$, we can construct $s_{j}$ similarly. Since successive $s_{j}$ differ only within the shrinking sets $N_{n j}$, they converge uniformly to a continuous surjection $f_{n+1}$, and $f_{n+1}^{-1}\left(p_{n}\right)=q_{n+1}$. To secure that the $f_{n}$ converge uniformly, it suffices to require that $(\alpha)$ the sum of the diameters of all $N_{n 1}$ is finite. Then $g=\lim f_{n}$ is a continuous surjection. Now the early $q_{i}, i \leqq n$, were $f_{n}^{-1}\left(p_{i-1}\right)$. If $(\beta)$ the diameters of the sets $N_{n 1}$ are sufficiently small fractions of the distance from $p_{n}$ to $\left\{p_{0}, \cdots, p_{n-1}\right\}$, then we get $q_{i}=g^{-1}\left(p_{i-1}\right)$. Since $C$ is compact, $g$ is uniformly continuous. Since $g$ is surjective, $g(Q)$ is dense. Ultrafilters in $Q$ having no limit in $Q$ have limits in $C-Q$, and $g$ takes them to nonconvergent ultrafilters in $g(Q)$; so $g \mid Q$ is a topological embedding.

M. K. Fort showed [1] that any two countable dense subsets $D, E$ of $H$ are equivalent by an autohomeomorphism of $H$. Thus there is, up to isomorphism, a unique uniform space $\rho Q$ which is homeomorphic with $Q$ and has completion homeomorphic with $H$. We have proved:

THEOREM 1. Every uniform space homeomorphic with $Q$ admits a uniformly continuous homeomorphism upon $\rho Q$. 
Shifting away from statements "up to isomorphism", $Q$ has many uniformities $\rho$ with completion $H$, and the result is that for each uniformity $\mu$ there is a coarser $\rho$. What other equivalence classes (under autohomeomorphism) of uniformities $\sigma$ have that property? Evidently, those which are coarser than some $\rho$. Such a uniformity $\sigma$ is again precompact. The completion of $\sigma Q$ is a quotient space of $H$ by a decomposition in which each single point of $Q$ is an equivalence class. Conversely, the uniformity induced by such a quotient space is coarser than some $\rho$.

TheOREM 2. For Peano continua, the property that the set of local cut points is of the first category is preserved by continuous surjections whose restriction to a dense set is homeomorphic.

Proof. Let $f: K \rightarrow L$ be such a mapping. $L$ is also a Peano continuum. By Lavrentiev's theorem, $f$ maps a dense $G_{\delta}$ set $D$ homeomorphically. Removing a first category $F_{\sigma}$ set containing the local cut points, we have a dense $G_{\delta}$ set $E$ of nonlocal cut points mapped homeomorphically. Since $E$ is dense and $K$ compact, $E$ is $f^{-1} f(E)$, and $L-f(E)$ is first category. In $L$, a connected neighborhood of a point $f(e)$ cannot be partitioned into $f(e)$ and two open sets $V_{1}, V_{2}$. For the inverse image would partition accordingly into $e, U_{1}, U_{2}$. Then $e$ is not in the closures of both $U_{i}$. But $f\left(U_{i}^{-}\right)$is compact and must contain $f(e)$.

Evidently a decomposition required to be homeomorphic on a dense set can do almost anything on a first category set. For instance, for $Q \subset H$, one can find a disjoint sequence of boundaries of neighborhoods of its points, with diameters converging to 0 ; this yields a decomposition of $H$ homeomorphic on $Q$ with the quotient one-dimensional at each point of $Q$.

2. Incompatible case. A separated uniformity on $Q$, compatible with the topology or not, but coarser than $\rho$, must be precompact; the completion is a quotient of $H$ by a mapping injective on $Q$. Since it is a quotient of $H$, it is a Peano continuum. Conversely:

THeorem 3. For every countable dense set $D$ in a Peano continuum $K$ there is a continuous map $H \rightarrow K$ taking $Q$ bijectively to $D$.

Proof. First, there is a continuous surjection $f_{0}: I \rightarrow K$. Since $K$ is locally arcwise connected and $D$ is dense in $K$, a sequence of small modifications $\left(f_{n}\right.$ to $\left.f_{n+1}\right)$ will converge to $g: I \rightarrow K$ such that $g^{-1}(D)$ is dense. Keeping each $f_{n}$ surjective, $g$ will be surjective. One can secure also that $g$ is not constant on any interval. Then the inverse image of any finite set is nowhere dense; hence $g^{-1}(D)$ contains a dense set on which $g$ is injective, and a larger dense set $A$ mapped bijectively to $D$.

Evidently two countable dense sets in $I$ containing the same number of end points are equivalent by a homeomorphism of $I$. Mrowka showed [3] 
that there is a map $H \rightarrow I$ mapping $Q \subset H$ injectively. It is easy to see that one can prescribe the number of endpoint values; and the image of $Q$ must be dense.

THEOREM 4. A countable uniform space is a bijective uniformly continuous image of every countable dense-in-itself uniform space if and only if its completion is a Peano continuum.

Proof. As remarked before Theorem 3, a bijective uniformly continuous image of $\rho Q$ has a Peano continuum for completion. Given such a space $\lambda D$ with completion $K$, given a countable dense-in-itself space $\mu X$, evidently $\mu$ contains a separated metric uniformity $\nu$. Since $\mu X$ is dense-initself, so is $\nu X$; thus it is homeomorphic with $Q$. Then Theorem 1 and Theorem 3 yield a uniformly continuous bijection $v X \rightarrow \lambda D$.

\section{REFERENCES}

1. M. K. Fort, Jr., Homogeneity of infinite products of manifolds with boundary, Pacific J. Math. 12 (1962), 879-884. MR 26 \#3030.

2. J. R. Isbell, Uniform spaces, Math. Surveys, no. 12, Amer. Math. Soc., Providence, R.I., 1964. MR 30 \#561.

3. S. Mrowka, Continuous functions on countable subspaces, Portugal. Math. 29 (1970), 177-180.

Department of Mathematics, State University of New York at Buffalo, AMHERST, New YORK 14226 